\title{
Injectable antimicrobial conductive hydrogels for wound disinfection and
}

\section{infectious wound healing}

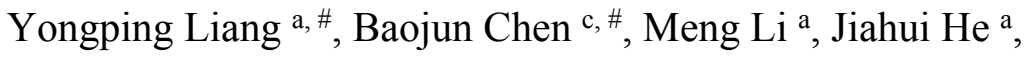
Zhanhai Yin ${ }^{\mathrm{c}}$, Baolin Guo ${ }^{\mathrm{a}, \mathrm{b}, *}$

a Frontier Institute of Science and Technology, and State Key Laboratory for Mechanical Behavior of Materials, Xi'an Jiaotong University, Xi'an, 710049, China

${ }^{b}$ Key Laboratory of Shaanxi Province for Craniofacial Precision Medicine Research, College of Stomatology, Xi'an Jiaotong University, Xi'an 710049, China

${ }^{c}$ Department of Orthopaedics, The First Affiliated Hospital, College of Medicine, Xi'an Jiaotong University, Xi'an, 710061 China

* To whom correspondence should be addressed.

E-mail: baoling@mail.xjtu.edu.cn

\# contributed equally to this work 


\section{Materials and methods}

\section{Synthesis of glycidyl methacrylate functionalized quaternized chitosan (QCSG)}

$1 \mathrm{~g}$ of chitosan ( $\mathrm{J} \& \mathrm{~K}$ Chemical, $\mathrm{Mn}=100,000-300,000 \mathrm{Da}$ ) was suspended in $36 \mathrm{~mL}$ of deionized water, and then $180 \mu \mathrm{L}$ of glacial acetic acid (Sigma-Aldrich) was added to the suspension. After stirring at $55{ }^{\circ} \mathrm{C}$ for $30 \mathrm{~min}, 2$ equivalent glycidyltrimethylammonium chloride (GTMAC, Sigma-Aldrich, relative to the amino group on the chitosan) was added to the chitosan-glacial acetic acid mixture under continuous stirring. The reaction mixtures were stirred at $55^{\circ} \mathrm{C}$ for $15 \mathrm{~h}$. Then, glycidyl methacrylate (GMA, Sigma-Aldrich) was added dropwise to the above reaction mixtures with continuous stirring at $55{ }^{\circ} \mathrm{C}$, respectively. The molar ratio of GMA to amino groups on the pure chitosan backbone was fixed at $0.5: 1.0$, and the reaction was performed for another $15 \mathrm{~h}$ at $55{ }^{\circ} \mathrm{C}$ in the dark condition. After the reaction, the undissolved polymer was removed by centrifuging the mixture at $5000 \mathrm{rpm}$ for $20 \mathrm{~min}$ at room temperature. The supernatant liquid was precipitated into pre-cooled acetone to obtain the crude product. For purifying the product, the crude product was dissolved in DI water, and then dialyzed exhaustively (MWCO 3500) against deionized water for three days in the dark condition. The pure product was obtained by lyophilization. The quaternary amination degrees of the QCSG copolymers were determined by titrating the content of chlorine ion. The chemical structure of QCSG was confirmed by ${ }^{1} \mathrm{H}$ NMR.

\section{Synthesis of gelatin methacrylate (GM)}


To produce the GM, $10 \mathrm{~g}$ of gelatin was dissolved in phosphate buffered saline (PBS) $(10 \% \mathrm{w} / \mathrm{v})$ at $50{ }^{\circ} \mathrm{C}$. Methacrylic anhydride $(0.8 \mathrm{~mL}$ per gram of gelatin) was added and the mixture was stirred for $3 \mathrm{~h}$ at $50{ }^{\circ} \mathrm{C}$. Then, the solution was diluted with PBS (5X in volume). The solution was dialyzed (MWCO $10 \mathrm{kDa}$ ) in distilled water for 7 days at $40{ }^{\circ} \mathrm{C}$ to remove unreacted methacrylic anhydride. Finally, the solution was lyophilized to obtain a dried GM foam. The degree of methacrylate modification was determined by NMR spectroscopy following a previously reported method. ${ }^{1}$

\section{Characterizations}

${ }^{1} \mathrm{H}$ NMR spectra of gelatin methacryloyl (GM) were performed using a Bruker Ascend $400 \mathrm{MHz}$ NMR instrument with deuteroxide serving as the solvents.

The morphologies of freeze-dried hydrogels were examined by a field emission scanning electron microscope (FE-SEM; QUTAN FEG 250, FEI) after sprayed with a thin gold layer. NIH Image J software was used to measure the pore diameters of hydrogel samples. For each hydrogel sample (at least 3 samples), every hydrogel had four pictures taken from various regions of the hydrogel. Every picture was measured by more than five pores.

\section{Swelling test}

Swelling test was used to determine the equilibrium swelling ratio (ESR) and stability of the hydrogels. The completely gelled wet hydrogels were put into $20 \mathrm{~mL}$ PBS $(0.01$ $\mathrm{M} \mathrm{pH}$ 7.4) in sealed vials at $37^{\circ} \mathrm{C}$ with shaking at $100 \mathrm{rpm}$. The test was not finished until the weight of all hydrogel kept constant. Following that, the hydrogels were weighed. ESR was calculated using the following equation: $E S R=\left(\mathrm{W}_{t}-\mathrm{W}_{\mathrm{i}}\right) / \mathrm{W}_{\mathrm{i}}$, where 
$\mathrm{W}_{\mathrm{i}}$ and $\mathrm{W}_{\mathrm{t}}$ represented the initial dry weight and after swelling weight, respectively. ${ }^{2}$

\section{Rheological property of the hydrogels}

The rheological test of these hydrogels were carried out by using a TA rheometer (DHR-2). Time sweep test with $1 \%$ constant strain and a constant frequency of $10 \mathrm{rad} / \mathrm{s}$ at $37^{\circ} \mathrm{C}$ was used to evaluate the stiffness of these hydrogels. $350 \mu \mathrm{L}$ of the polymer mixture was placed between $20 \mathrm{~mm}$ parallel plates with a gap of $1000 \mu \mathrm{m}$ and the periphery was sealed by silicone oil to prevent the evaporation of water. ${ }^{3}$

\section{Mechanical properties of GT-DA/CS/CNT hydrogel}

Hydrogel samples were prepared into cylindrical shape $(\sim 8 \mathrm{~mm}$ high $\times 10 \mathrm{~mm}$ in diameter) for compression test at $25{ }^{\circ} \mathrm{C}$. The compression test was conducted using a rheometer (Model DHR-2, TA Instruments) with the compression strain up to $60 \%$ at a strain speed of $6 \mathrm{~mm} / \mathrm{min}$. All these tests were employed more than 3 times ${ }^{4}$.

\section{Conductivity test}

Before performing the conductivity test, hydrogels were swollen in deionized water and the excess water on the hydrogel surface was removed using filter paper. Followed that, the hydrogel was transferred into a wafer after exposed to air for $30 \mathrm{~min}$. The conductivity of the hydrogels was measured by employing a digital 4-probe tester measurements. $^{5}$

\section{Photo-thermal effect of the hydrogels}

In order to elucidate the hydrogels' photo-thermal effect, the as-prepared hydrogel was cut into cubes $(5 \times 5 \times 5 \mathrm{~mm})$ and then exposed to an NIR laser (MDL-III-808nm1000mW, Changchun New Industries Optoelectronics Tech Co., Ltd.) at a power 
density of $1.0 \mathrm{~W} / \mathrm{cm}^{2}$ for $10 \mathrm{~min}$. The heat maps and temperature profiles of the hydrogels were recorded using an infrared (IR) thermal camera.

\section{In vitro drug release study}

$200 \mu \mathrm{L}$ of QCSG/GM/GO1 hydrogels loaded with desired amount of doxycycline (2 $\mathrm{mg} / \mathrm{mL}$ ) were prepared in a $4 \mathrm{~mL}$ centrifuge tube. After allowing complete gelation, the hydrogels formed and the drug was encapsulated in the hydrogels in situ. $3 \mathrm{~mL}$ of PBS with $\mathrm{pH}$ values of 7.4 was pipetted into each tube with shaking speed of $100 \mathrm{rpm}$ at $37^{\circ} \mathrm{C}$. After a predetermined time, $1 \mathrm{~mL}$ of the release buffer was removed for further analysis. Subsequently, $1 \mathrm{~mL}$ of fresh buffer was added to the tube in order to maintain a constant volume. ${ }^{6}$ The concentrations of the drugs released from hydrogels were analyzed by the UV-vis spectrophotometer (PerkinElmer Lambda 35). The $\lambda_{\max }$ of doxycycline was $351 \mathrm{~nm}$.

\section{NIR irradiation enhanced in vitro antibacterial performance of hydrogels}

The QCSG/GM/GO1 hydrogel cubes $(5 \times 5 \times 5 \mathrm{~mm})$ were prepared by using sterilized QCSG, GM solutions and GO suspensions under a sterile environment, and then equilibrated with sterilized Dulbecco's phosphate buffered saline (DPBS). $10 \mu \mathrm{L}$ of bacterial suspension in sterilized DPBS $\left(10^{8} \mathrm{CFU} / \mathrm{mL}\right)$ was added onto the surface of the hydrogel cubes. Then, the hydrogel was exposed to NIR laser light (808 nm, 1.0 $\mathrm{W} / \mathrm{cm}^{2}$ ) for varying periods from 0 to $1,3,5$ and $10 \mathrm{~min}$, respectively. After allowing all the groups to contact with bacteria for preset time, $1 \mathrm{~mL}$ of sterilized DPBS was added into each well to re-suspend any bacterial survivor. Then, $10 \mu \mathrm{L}$ of the above bacterial survivor resuspension was diluted and added onto agar plate, and the colony- 
forming units on the agar plate were counted after incubated for 18 to $24 \mathrm{~h}$ at $37^{\circ} \mathrm{C}$. $^{7}$ Tests were repeated three times for each group and the results were expressed as killing $\%$ :

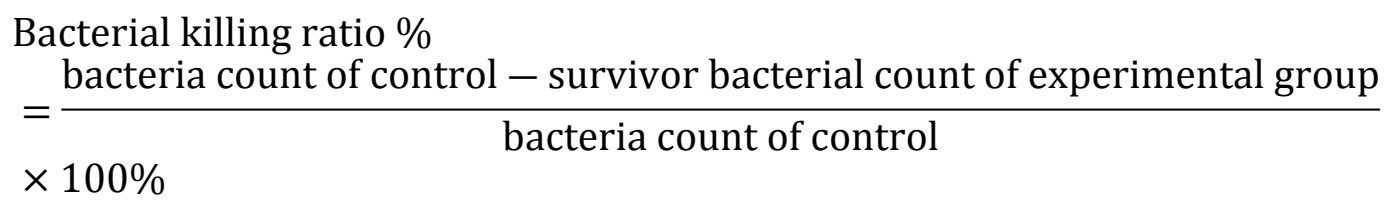

\section{Inhibition zone test of antibiotics loaded QCSG/GM/GO hydrogel}

In this test, agar plates were inoculated with $100 \mu \mathrm{L}$ of the bacterial suspension $\left(10^{8}\right.$ $\mathrm{CFU} / \mathrm{mL}$ ). The samples were exposed to bacteria on solid media (nutrient agar), and the inhibition zone around each sample was measured and recorded as the antibacterial effect of doxycycline loaded QCSG/GM/GO1 hydrogel. Hydrogels were placed on the agar plate and incubated at $37^{\circ} \mathrm{C}$ for $24 \mathrm{~h}$. The inhibition zone for bacterial growth was detected visually. After $24 \mathrm{~h}$, the hydrogels were transferred to a new agar plate covered with bacteria and incubated for another $24 \mathrm{~h}$. Subsequently, the above operation was repeated again until no inhibition zone appeared on the new agar plate. ${ }^{8}$

\section{Hemolytic activity test of the hydrogels}

Hemolysis activity assay was conducted according to reference ${ }^{9}$. Erythrocytes were separated by centrifugation (at 1,000 rpm) from the mice blood for 10 minutes. The obtained erythrocytes were washed three times with Tris buffer and then diluted to a final concentration of $5 \%(v / v)$. Hydrogel $(500 \mu \mathrm{L})$ with erythrocytes stock $(500 \mu \mathrm{L})$ was added to a 24 -well microplate, then shaken in an incubator at $37^{\circ} \mathrm{C}$ for $1 \mathrm{~h}$ with a shaking speed of $150 \mathrm{rpm}$. After that, the microplate well contents were centrifuged (at $1,000 \mathrm{rpm})$ for 10 minutes and the supernatant $(100 \mu \mathrm{L})$ was then introduced into a 96- 
well microplate. The absorbance of the solution was read at $540 \mathrm{~nm}$ by a microplate reader (Molecular Devices). 0.1\% Triton $\mathrm{x}-100$ was used as the positive control while Tris buffer was used as the negative control. The hemolysis percentage was calculated from the relation: Hemolysis $(\%)=\left[\left(\mathrm{A}_{\mathrm{p}}-\mathrm{A}_{\mathrm{b}}\right) /\left(\mathrm{A}_{t}-\mathrm{A}_{\mathrm{b}}\right)\right] \times 100 \%$ where $\mathrm{A}_{\mathrm{p}}$ was the absorbance value for the experiment group. $A_{t}$ was the absorbance value for the Triton $\mathrm{x}-100$ positive control and $\mathrm{A}_{\mathrm{b}}$ was the absorbance value for Tris buffer.

\section{Cytocompatibility evaluation of the hydrogels}

The cytotoxicity of hydrogels was measured by employing a direct contact test between hydrogels and L929 cells. As described before in the section of hydrogel preparation, the solution of QCSG, GM and GO were sterilized at $60{ }^{\circ} \mathrm{C}$ for $12 \mathrm{~h}$. the solution of APS and TEMED was sterilized by filtration ( $0.22 \mu \mathrm{m}$ filter, Millipore). After mixing, the mixture was poured into a sterile Petri dish to form a hydrogel film with about 1.5 mm thickness at $37{ }^{\circ} \mathrm{C}$ in $\mathrm{CO}_{2}$ incubator. Then hydrogel film was cut into $5 \mathrm{~mm}$ diameter disks using a Harris Micro-Punch (Harris Uni-Core ${ }^{\mathrm{TM}}$, USA) and the dulbecco's modified eagle medium (DMEM) (Gibco) supplemented with 10\% fetal bovine serum (Gibco), $1.0 \times 10^{5} \mathrm{U} / \mathrm{L}$ penicillin (Hyclone) and $100 \mathrm{mg} / \mathrm{L}$ streptomycin (Hyclone) was used as the complete growth medium. L929 cells were seeded in 96well plate at a density of 20000 cells/well. After cultured for $24 \mathrm{~h}$, the hydrogel disks were introduced into the wells. The cell proliferation and viability under the hydrogel was evaluated by alamarBlue ${ }^{\circledR}$ assay and LIVE/DEAD ${ }^{\circledR}$ Viability/Cytotoxicity Kit assay after $24 \mathrm{~h}$. The hydrogel disks and medium were removed and $10 \mu \mathrm{L}$ of alamarBlue ${ }^{\circledR}$ reagent in $100 \mu \mathrm{L}$ complete growth medium was then added into each 
well. The plate was incubated for $4 \mathrm{~h}$ in a humidified incubator containing $5 \% \mathrm{CO}_{2}$ at $37^{\circ} \mathrm{C}$. After that, $100 \mu \mathrm{L}$ of the medium in each well was transferred into a 96-well black plate (Costar). Fluorescence was read using $560 \mathrm{~nm}$ as the excitation wavelength and $600 \mathrm{~nm}$ as the emission wavelength using a microplate reader (Molecular Devices) according to the manufacturer's instructions. Cells seeded on TCP without hydrogel disc served as the positive control group. Tests were repeated four times for each group. Cell adhesion and viability were observed under an inverted fluorescence microscope (IX53, Olympus). The cell viability was evaluated by alamarBlue ${ }^{\circledR}$ assay after cultured for $24 \mathrm{~h} .{ }^{10}$

\section{Histocompatibility test}

Animal experiments were performed according to the guide for the care and use of laboratory animals, established by the committee on animal research at Xi'an Jiaotong University. Following a general anesthesia, QCSG/GM/GO hydrogel pieces were subcutaneously implanted on the back of the female SpragueDawley (SD) rats that were 200-250 $\mathrm{g}$ in weight ( $\mathrm{n}=4$ per group). After surgery, the rats were permitted free access to water and food to recover from the surgery. After 1 week and 4 weeks, the rats were sacrificed, and the implanted films and surrounding tissues were carefully dissected for histological staining to evaluate the inflammatory responses of QCSG/GM/GO in rats. Tissues were embedded in paraffin, sectioned ( $3 \mathrm{~mm}$ thick), and stained by hematoxylin and eosin $(\mathrm{H} \& \mathrm{E})$, respectively. ${ }^{11}$

\section{In vivo wound healing evaluation with an infected full-thickness skin defect model}

To further evaluate the promoting effect of QCSG/GM/GO hydrogel on wound repair, 
MRSA infected full-thickness skin defect model was established. Our animal experiments were approved by the institutional review board of Xi'an Jiaotong University. Female Kunming mice weighting 25-35 g and 5-6-week age were used for studies. All mouse was acclimatized for 1 week before surgery and then randomly divided into 4 groups including Tegaderm ${ }^{\mathrm{TM}}$ film (control), QCSG/GM/GO0 hydrogel, QCSG/GM/GO1 hydrogel and QCSG/GM/GO1/Doxy hydrogel. After anesthesia and shaved, $7 \mathrm{~mm}$ diameter skin wounds were created and $50 \mu \mathrm{L}$ Methicillin-resistant Staphylococcus aureus $\left(10^{8} \mathrm{CFU} / \mathrm{mL}\right)$ was used to establish infection after $48 \mathrm{~h}$ implantation. Then, control wounds were added with $50 \mu \mathrm{L}$ of PBS and dressed with Tegaderm ${ }^{\mathrm{TM}}$ film (3M Health Care, USA), hydrogel group wounds were added with 50 $\mu \mathrm{L}$ of $\mathrm{QCSG} / \mathrm{GM} / \mathrm{GO} 0, \mathrm{QCSG} / \mathrm{GM} / \mathrm{GO} 1$ or $\mathrm{QCSG} / \mathrm{GM} / \mathrm{GO} 1 / \mathrm{Doxy}$ hydrogel. All tissues were collected on each 5 mice in 4 groups on the $3^{\text {rd }}, 7^{\text {th }}$, and $14^{\text {th }}$ day. All samples were stored at $-80^{\circ} \mathrm{C}$ before analysis. The regeneration process of wounds was assessed by wound closure ratio. ${ }^{12}$ For the biochemical analysis, on the $3^{\text {rd }}, 7^{\text {th }}$, and $14^{\text {th }}$ day, the samples were collected and made into disc-shaped tissue $($ diameter $=1 \mathrm{~cm})$, and collagen amount was evaluated by Image-Pro Plus from Masson trichrome staining. All operations were followed manufacturer's instruction. ${ }^{13}$

For wound closure monitoring, on the $3_{\mathrm{rd}}, 7^{\text {th }}, 14^{\text {th }}$ day, the mice in each group were performed standard anesthesia, then wound closure were measured by Image J.

Wound closure (\%) were calculated using the formula below:

Wound closure $=($ area $(0$ day $)-$ area $($ day $)) /($ area $(0$ day $)) \times 100 \%$, where " $n$ " represents the day, such as 3 th, 7th, and 14th day. All results were analyzed 
by one-way ANOVA test.

\section{Histology and immunohistochemistry}

For evaluation of epidermal regeneration and inflammation in wound area, samples collected on the $3^{\text {rd }}, 7^{\text {th }}$, and $14^{\text {th }}$ day were fixed with $4 \%$ paraformaldehyde for $1 \mathrm{~h}$, then embedded in paraffin and cross sectioned to $4 \mu \mathrm{m}$ thickness slices, and then stained with Haematoxylin-Eosin (Beyotime, China). All slices were analyzed and photocaptured by microscope ((IX53, Olympus, Japan). The regenerated skins from the wound site were also excised on 3, 7, and 14 days for immunofluorescence staining. The fixed and frozen sections were stained with Anti-VEGF antibody (abcam) and IL6 (Affinity Biosciences), respectively. FITC-conjugated goat anti-mouse IgG (cwbiotech) and FITC-conjugated goat anti-rabbit IgG (cwbiotech) were used as the secondary antibody to reveal VEGF and IL-6 expression. The nuclei were stained with DAPI containing mounting solution. Slides were observed under an inverted fluorescence microscope (IX53, Olympus).

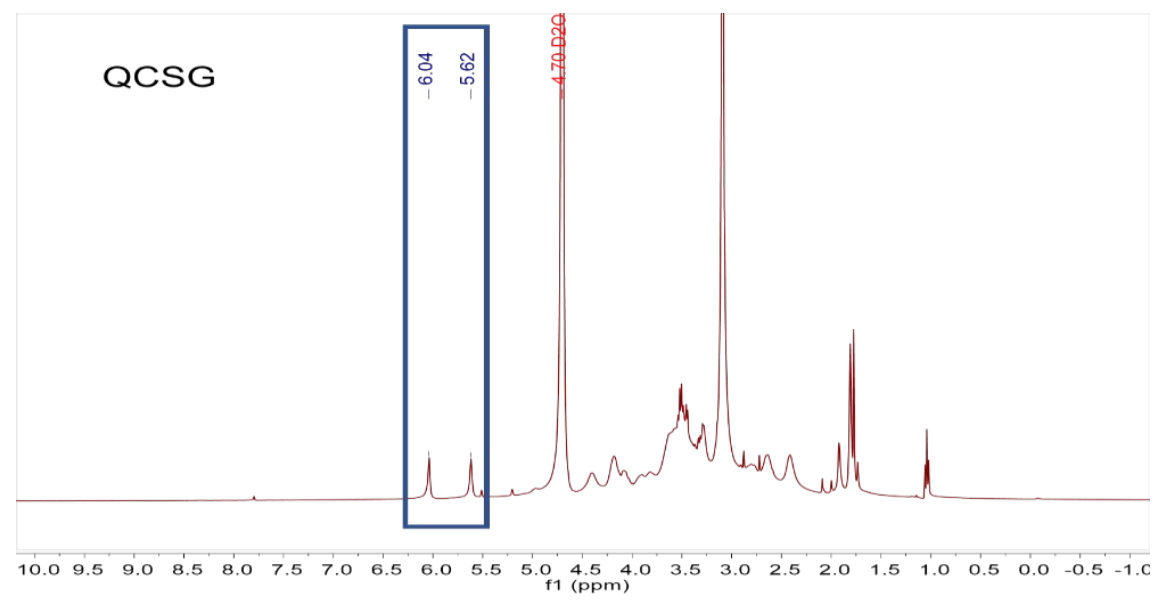

Figure S1. ${ }^{1} \mathrm{H}-\mathrm{NMR}$ spectra of QCSG 


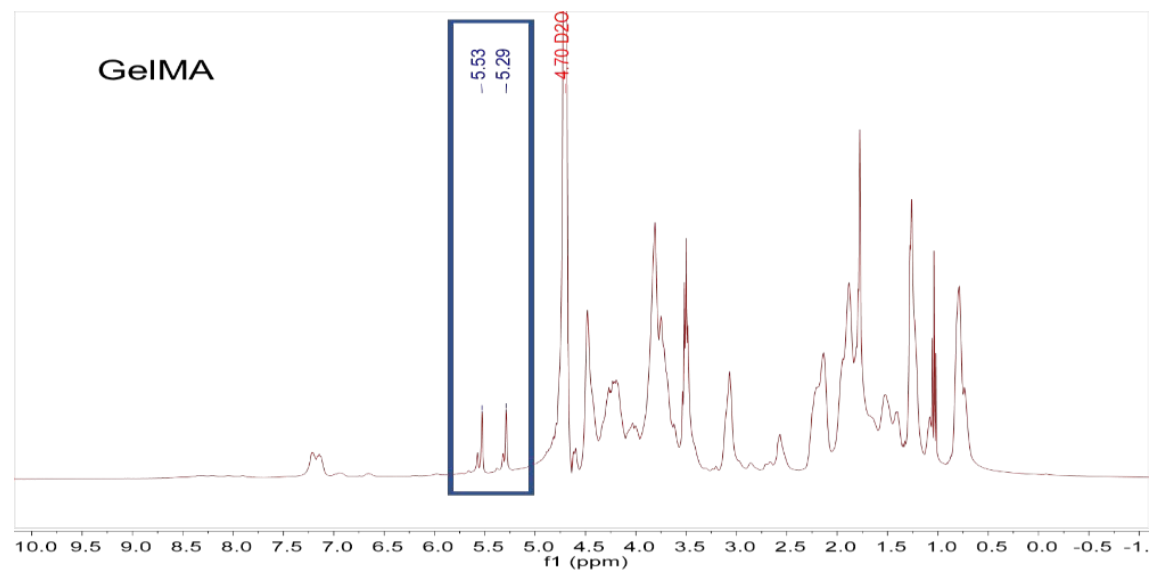

Figure S2. ${ }^{1} \mathrm{H}-\mathrm{NMR}$ spectra of GM
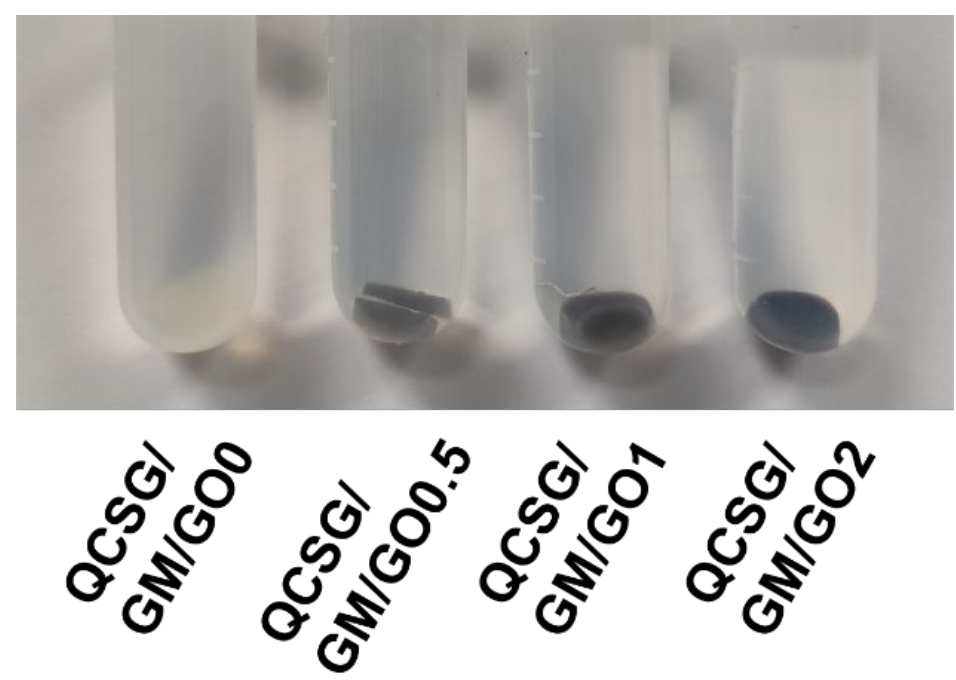

Figure S3. Hydrogels were soaked in PBS for 3 days

\section{References}

(1) Chen, Y.-C.; Lin, R.-Z.; Qi, H.; Yang, Y.; Bae, H.; Melero-Martin, J. M.;

Khademhosseini, A. Functional Human Vascular Network Generated in Photocrosslinkable Gelatin Methacrylate Hydrogels. Adv. Funct. Mater. 2012, 22, 2027-2039.

(2) Zhao, X.; Dong, R.; Guo, B.; Ma, P. X. Dopamine-Incorporated Dual Bioactive 
Electroactive Shape Memory Polyurethane Elastomers with Physiological Shape Recovery Temperature, High Stretchability, and Enhanced C2C12 Myogenic Differentiation. ACS Applied Materials \& Interfaces 2017, 9, 29595-29611.

(3) Qu, J.; Zhao, X.; Ma, P. X.; Guo, B. Injectable antibacterial conductive hydrogels with dual response to an electric field and $\mathrm{pH}$ for localized "smart" drug release. Acta Biomater 2018, 72, 55-69.

(4) Zhao, X.; Guo, B.; Wu, H.; Liang, Y.; Ma, P. X. Injectable antibacterial conductive nanocomposite cryogels with rapid shape recovery for noncompressible hemorrhage and wound healing. Nat. Commun. 2018, 9, 2784.

(5) Deng, Z.; Guo, Y.; Zhao, X.; Ma, P. X.; Guo, B. Multifunctional stimuli-responsive hydrogels with self-healing, high conductivity, and rapid recovery through host-guest interactions. Chem. Mater. 2018, 30, 1729-1742.

(6) Tormos, C. J.; Abraham, C.; Madihally, S. V. Improving the stability of chitosangelatin-based hydrogels for cell delivery using transglutaminase and controlled release of doxycycline. Drug Deliv. Transl. Re. 2015, 5, 575-584.

(7) Wu, M.-C.; Deokar, A. R.; Liao, J.-H.; Shih, P.-Y.; Ling, Y.-C. Graphene-based photothermal agent for rapid and effective killing of bacteria. ACS Nano 2013, 7, 12811290.

(8) Ryu, S. J.; Jung, H.; Oh, J. M.; Lee, J. K.; Choy, J. H. Layered double hydroxide as novel antibacterial drug delivery system. J. Phys. Chem. Solids 2010, 71, 685-688.

(9) Sasidharan, A.; Panchakarla, L. S.; Sadanandan, A. R.; Ashokan, A.; Chandran, P.; Girish, C. M.; Menon, D.; Nair, S. V.; Rao, C.; Koyakutty, M. Hemocompatibility and 
macrophage response of pristine and functionalized graphene. Small 2012, 8, 12511263.

(10) Zhao, X.; Dong, R.; Guo, B.; Ma, P. X. Dopamine-incorporated dual bioactive electroactive shape memory polyurethane elastomers with physiological shape recovery temperature, high stretchability, and enhanced c2c12 myogenic differentiation. ACS Appl. Mater. Interfaces 2017, 9, 29595-29611.

(11) Wu, Y.; Wang, L.; Zhao, X.; Hou, S.; Guo, B.; Ma, P. X. Self-healing supramolecular bioelastomers with shape memory property as a multifunctional platform for biomedical applications via modular assembly. Biomaterials 2016, 104, $18-31$

(12) Fan, Z.; Liu, B.; Wang, J.; Zhang, S.; Lin, Q.; Gong, P.; Ma, L.; Yang, S. A novel wound dressing based on ag/graphene polymer hydrogel: effectively kill bacteria and accelerate wound healing. Adv. Funct. Mater. 2014, 24, 3933-3943.

(13) Qu, J.; Zhao, X.; Liang, Y.; Zhang, T.; Ma, P. X.; Guo, B. Antibacterial adhesive injectable hydrogels with rapid self-healing, extensibility and compressibility as wound dressing for joints skin wound healing. Biomaterials 2018, 183, 185-199. 